\title{
Design of Light Trapping Nanopatterned Solar Cells Based on Three-Dimensional Optical and Electrical Modeling
}

\author{
Hui-Hsin Hsiao, ${ }^{1}$ Hung-Chun Chang, ${ }^{1}$ and Yuh-Renn $\mathrm{Wu}^{1, *}$ \\ ${ }^{1}$ Graduate Institute of Photonics and Optoelectronics, National Taiwan University, Taipei, Taiwan 10617. \\ *Corresponding author: yrwu@ntu.edu.tw
}

\begin{abstract}
The optical and electrical properties of a new type photonic-plasmonic nanostructure on the back contact of solar cells were investigated numerically through the threedimensional (3D) finite-difference time-domain (FDTD) method and the Poisson and drift-diffusion (DDCC) solver. The focusing effect and the Fabry-Perot resonances are identified as the main mechanisms for the enhancement of the optical generation rate as well as the short circuit current density. In addition, the surface topography of the nanopattern has a strong effect on the device physics such as the potential and recombination profiles, and therefore influencing the electrode collecting efficiency of the photocurrents.
\end{abstract}

\section{Index Terms - solar cell, light trapping.}

\section{INTRODUCTION}

Thin film solar cells offer the benefits of reduced materials and fabrication costs and are attractive candidates for lightweight and flexible devices. To achieve efficient absorption of the solar spectrum with thin semiconductor volumes, many researches focus on improving light trapping schemes, which are frequently realized by random or periodic textures [1]. In this research, we propose a new type photonicplasmonic nanostructure called anti-ring array comprised of a nanodome centered inside a nanohole, as the back reflector geometry for a-Si:H solar cells. Then, we use our in-house developed three-dimensional (3-D) finite-difference timedomain (FDTD) program combined with 3-D Poisson and drift-diffusion (DDCC) [2] solver to analyze the performance of the proposed cell. The fully 3D model enables us to systematically study the influences of the anti-ring back reflector in both optical and electronic properties. The optimization of optical scattering especially in the long wavelength range and the influence of surface and interface recombination will all be studied in this work without too many approximations such as $2 \mathrm{D}$ or $1 \mathrm{D}$ work.
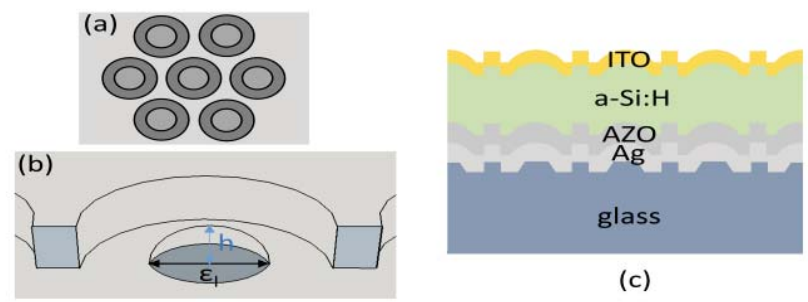

(c)

Fig. 1. The schematic (a) top view and (b)(c) cross-section view of the anti-ring array.

\section{OPTICAL GENERATION}

Fig. 1 shows the schematic view of the anti-ring structure, where the hexagonal anti-ring nanopattern is first fabricated on the surface of the glass substrate. An 120-nm-thick silver film and a 160-nm-thick ZnO:Al (AZO) layer are then deposited above the nano-patterned glass substrate. The $n-i-p$ a-Si:H layer with thickness $180 \mathrm{~nm}$ and indium tin oxide (ITO) layer with thickness $50 \mathrm{~nm}$ are deposited accordingly. To simulate the hexagonal anti-ring arrays, the triangular lattice periodic boundary condition (PBC) was used in the FDTD program, and the nanodomes inside the nanoholes with diameter $D_{h}$ were approximated by half-ellipsoids with the major axis $\varepsilon_{l}$ and semi-minor axis $h$ fixed to $100 \mathrm{~nm}$. We have designed a serious of samples with different pitch $P$ $(200,400,500,700,800,1000 \mathrm{~nm})$ and fixed the ratio of $\varepsilon_{l} / D_{h}$ to be roughly equal to 0.7 . In addition, the dielectric constant of the $\mathrm{SiO}_{2}$ was set at 1.47 , while those of other materials were modeled with Drude-Lorentz dispersion. The optical generation rate $G_{\text {opt }}(\lambda)$ was then calculated by

$$
G_{\text {opt }}(\lambda)=\frac{1}{\hbar \omega} \operatorname{Re}[\vec{\nabla} \cdot \vec{P}]=\frac{\varepsilon^{\prime \prime}|\vec{E}|^{2}}{2 \hbar}
$$

where $\vec{P}$ is the polarization, $\varepsilon^{\prime \prime}$ is imaginary part of the permittivity, and $\vec{E}$ is the electric field. By weighting the FDTD results with respect to the AM $1.5 \mathrm{G}$ spectrum, the solar spectrum weighted $G_{\text {opt }}$ is obtained. Fig. 2(a) shows the spectra of $G_{\text {opt }} / G_{\text {Sun }}$ for the flat and designed samples, where $G_{\text {Sun }}$ is the generation rate under full absorption condition. In the case of the flat cell, one can see a broad hump around $500 \mathrm{~nm}$ and a major peak around $670 \mathrm{~nm}$. The broad hump around $\lambda=500 \mathrm{~nm}$ originates from the maximal solar spectral intensity distribution around this wavelength, while the peak around $\lambda=670 \mathrm{~nm}$ is associated with the second order Fabry-Perot resonances within the a-Si layer (Fig. 3(a)). For nano-structured cells, the enhancement of $G_{\text {opt }}$ around the broad hump is largest for sample P200 compared with the flat reference, while prominent redshift peaks are shown for samples P200 to P1000 in the longer wavelength range. For example, one can see a major peak around $680 \mathrm{~nm}$ for sample P200. According to the modulus of the electric field, $|\boldsymbol{E}|$, distributions shown in Fig. 3(b), this peak is associated with the focusing effect of the nanodome shape. Once the focusing hot spot is concentrated within the active layer, the generation rate can be greatly enhanced. In 

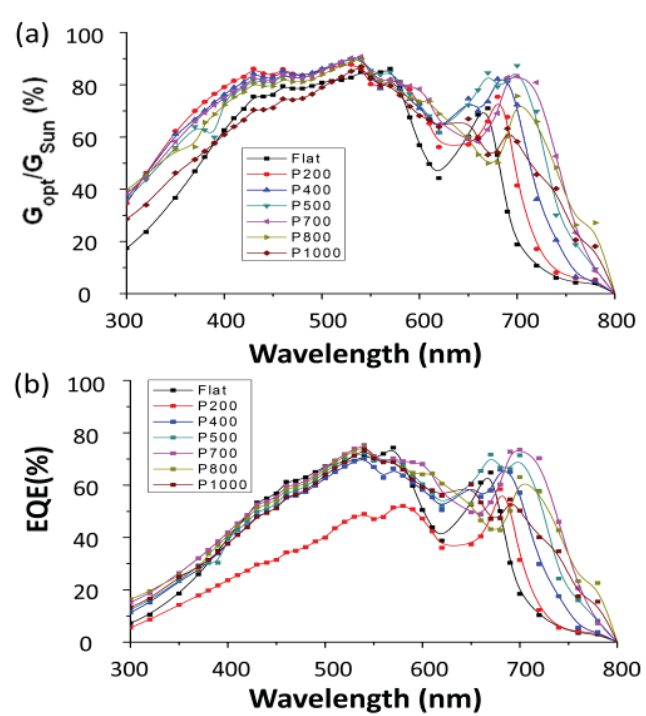

(c)

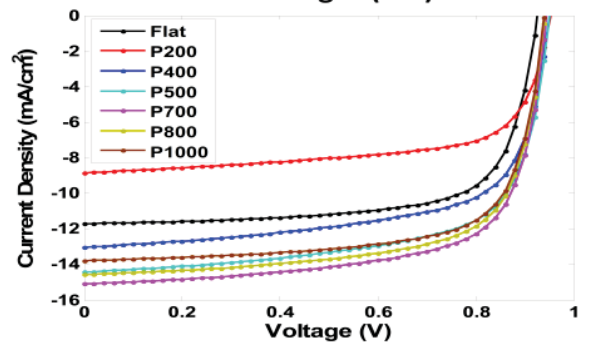

Fig. 2. The spectra of (a) $G_{\text {opt }} / G_{\text {Sun }}$ in $\%$ and (b) EQE for designed nanostructured cells and for the flat reference. This is calculated by considering the optical and electrical properties with 3D FDTD and DDCC. (c) The corresponding J-V curves.

addition, two peaks can be seen around $\lambda=650 \mathrm{~nm}$ and $\lambda=$ $685 \mathrm{~nm}$ for sample P400, and they redshift to $\lambda=670 \mathrm{~nm}$ and $\lambda=700 \mathrm{~nm}$, respectively, for sample P500. The shorter wavelength peak is caused by the aforementioned focusing effect, while the longer wavelength peak is associated with the second order Fabry-Perot resonances (not shown). For sample P700, these two peaks converge into one peak around $\lambda=710 \mathrm{~nm}$, and therefore having the optimal photocurrent among these samples.

\section{THE DYNOMICS OF CARRIERS TRANSPORT}

Next, the calculated $G_{\text {opt }}$ was implemented into our 3-D DDCC solver, where only the n-i-p layers were considered in analyzing the carrier transport phenomenon. The calculated external quantum efficiency (EQE) and the photocurrent density-voltage $(\mathrm{J}-\mathrm{V})$ curves for both the nanostructured cells and the flat reference are shown in Fig. 2(b) and Fig. 2(c), respectively. As we can see, the short circuit current $\left(J_{s c}\right)$ reaches the maximum value for sample P700, while different pitch nanostructures does not have significant effects on open circuit voltage $\left(V_{o c}\right)$.

In addition, one can also notice a surprising result that sample P200 has the smallest $J_{s c}$, even worse than the flat reference, and its EQE spectrum displays a significant reduction compared with other cells. We thus examined the non-radiative and radiative recombination profiles for these cells. Taking sample P1000 as a comparable example, we found that the non-radiative and radiative recombination profiles for sample P200 are not only stronger but also penetrating deeper into the i-Si layer than those for sample P1000 (Figs. 3(c) and 3(d)), especially near the nanodome. In view of such profiles, a question then naturally emerges: What causes the non-radiative and radiative recombination happened in the deep i-layer? According to the potential distributions for sample P200 and P1000 at 0V bias (not shown), the effective n-type flat band region for sample P200 is extended much deeper than that for sample P1000, which almost reaches to the middle of the i-layer. This is because the coverage of ITO electrode above the nanodome structure is similar to metallic-shell/dielectric-core structures. The metallic-shell will screen the outer potential fluctuation, thus making the potential uniform inside the core. The recombination loss thus increases owing to the extensive flat band region. Therefore, we should take into account not only the light trapping efficiency but also the photocurrent colleting efficiency when designing the optimistic texturing structures.

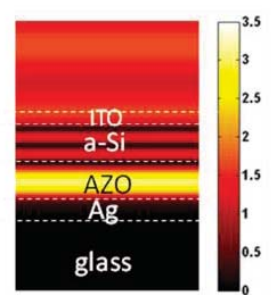

(a)

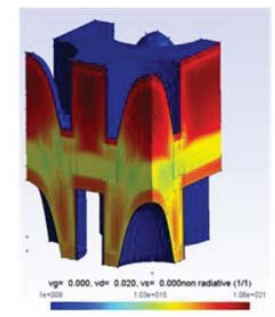

(c)

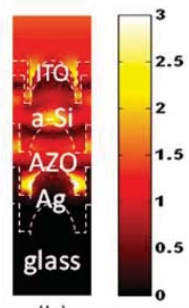

(b)

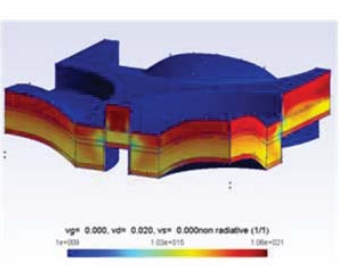

(d)
Fig. 3. The $|\boldsymbol{E}|$ distributions for (a) the flat reference at $\lambda=670 \mathrm{~nm}$ and (b) for sample P200 at $\lambda=680 \mathrm{~nm}$. The non-radiative recombination profiles for (c) sample P200 and (d) sample P1000.

\section{ACKNOWLEDGMENT}

This work was supported by National Science Council in Taiwan under grants NSC101-2221-E-002-147-MY2, NSC102-2811-E-002-050, and 102-2221-E-002 -194 -MY3..

\section{REFERENCES}

[1] V. E. Ferry, M. A. Verschuuren, M. C. v. Lare, R. E. Schropp, H. A. Atwater, and A. Polman, "Optimized spatial correlations for broadband light trapping nanopatterns in high efficiency ultrathin film a-Si: H solar cells," Nano Lett., vol. 11, pp. 4239-4245, 2011.

[2] C.-K. Li, H.-C. Yang, T.-C. Hsu, Y.-J. Shen, A.-S. Liu, and Y.-R. Wu, "Three dimensional numerical study on the effciency of a core-shell InGaN/GaN multiple quantum well nanowire light-emitting diodes," J. Appl. Phys., vol. 113, 183104, 2013. 